\title{
Temas y fuentes para la historia escolar en pueblos indígenas. Una reflexión desde los archivos de la Sierra Norte de Puebla, ca. 1876-1940
}

\author{
Ariadna Acevedo Rodrigo \\ Departamento de Investigaciones Educativas \\ Centro de Investigación y de Estudios Avanzados (Cinvestav) \\ aacevedo@cinvestav.mx
}

La historia escolar en México ha abordado la experiencia de poblaciones indígenas principalmente para el periodo colonial y el posrevolucionario, mientras que el tramo que va de la Independencia a la Revolución apenas empieza a ser estudiado. Tres libros recientes sobre el Estado de México desarrollaron a fondo el tema, pero para reconsiderar globalmente el papel de estas escuelas en la historia mexicana, son necesarias investigaciones sobre otros estados del país. ${ }^{2}$ Esta reseña considera el tipo de información que podemos encontrar en los archivos del estado de Puebla para contribuir a una historia escolar en pueblos indígenas durante los periodos del Porfiriato, Revolución y posrevolucionario (1876-1940).

Para abordar este tema se hace necesario tomar en cuenta los cambios históricos en la manera de concebir lo indígena. El historiador debe estar atento tanto a la manera en que el término se concebía en la época estudiada, como la forma en que se define en el presente del historiador, intentando evitar anacronismos, así que conviene empezar con algunas aclaraciones. Cuando hacemos referencia a la historia de la educación indígena frecuentemente no reparamos en cuál es el sentido que le damos al adjetivo "indígena". Estrictamente, la educación dirigida a la población indígena por el Estado mexicano en el siglo xx, a través de la Secretaría de Educación Pública (SEP), el Departamento Autónomo de Asuntos Indígenas

\footnotetext{
${ }^{1}$ De los trabajos sobre la colonia, entre los más recientes tenemos los de Tanck (1999) y Cedeño (2013), mientras que para el periodo 1920-1940 hay una amplia bibliografía sobre escuelas rurales federales que incluye localidades consideradas indígenas; por ejemplo, Vaughan, 2001; Rockwell, 2007; Civera, 2008; Manzanilla, 2015; Alfonseca, 2015; Flores, 2017. La historia de la educación para el periodo 1821-1876 se ha concentrado en historias generales para el conjunto del país y ha empezado a desarrollar una historia conceptual (Staples, 2005; Roldán, 2014). La escasez de trabajos sobre escuelas en poblaciones indígenas en el XIX ha sido señalada por Escalante (2003) y Sigüenza y Fabián (2013).

${ }^{2}$ Me refiero a Bazant (2002), Bustamante (2014) y Escalante (2014). Para Oaxaca ya hay capítulos, artículos y tesis de investigación pero aún no libros, por ejemplo, Traffano (2007 y 2016) y Martínez (2014). Véase también la revisión de Martín y Escalante (2017) sobre la bibliografía disponible.
} 
(DAAI) o el Instituto Nacional Indigenista (INI), puede describirse de manera más precisa como "indigenista" ( o "campesinista") en lugar de "indígena". Fue una política pública inicialmente diseñada por funcionarios que se consideraban no indígenas pero capaces de definir las necesidades de la población concebida como indígena.

El periodo 1876-1940 es interesante porque incluye cuatro décadas anteriores a la Revolución de 1910 y los regímenes emanados de ella. El campesinismo e indigenismo posrevolucionarios, al insistir en los logros de la Revolución, cerraron los ojos a la historia previa. Sin embargo, algunos de los estudios realizados a partir de la década de 1990 sobre las escuelas federales posrevolucionarias dejaron claro que para entender lo ocurrido en 1920-1940 era sumamente útil conocer la situación de la instrucción pública prerrevolucionaria (Vaughan, 2001; Rockwell, 2007; Giraudo, 2014: 173, 175). ${ }^{3}$ Si bien las investigaciones enfocadas exclusivamente en las escuelas del campo durante el siglo XIX siguen siendo escasas, lo que sí sabemos ya es que existían más escuelas de lo que habíamos creído hasta hace poco (Bazant, 2002; Escalante, 2014; Acevedo, 2015; Alfonseca, 2015; Chowning, 2017). La diferencia con el periodo posrevolucionario es que estas escuelas no respondían ni a una política explícitamente dirigida a indígenas ni a una creada específicamente para campesinos. La situación a lo largo del siglo XIX hasta el Porfiriato no debe sorprendernos. Al abolirse con la Independencia la categoría legal de "indio", en la cual se fundaba la existencia de repúblicas o pueblos de indios, con sus propias instituciones, las políticas dirigidas a la población dejaron de hacer una distinción legal entre indios y no indios para dirigirse a todos como ciudadanos. La política liberal de trato igual buscó romper con las distinciones coloniales y provocó una serie de cambios políticos cruciales. ${ }^{4}$ Esto trajo, además, un cambio considerable en la información que puede encontrarse en las fuentes consultadas por el historiador. Aun así, la desaparición de la categoría legal no necesariamente implicó la desaparición de los usos sociales del término. En los casos de Cuetzalan y Huehuetla (Sierra Norte de Puebla) durante el Porfiriato ya no solían utilizarse en la documentación oficial los términos "indio" o "indígena", ni el de "gente de razón" para los no indios; tenemos pistas, sin embargo, de que siguieron teniendo usos sociales y más adelante mencionaré algunas de las diferencias existentes en cuanto a distribución de la población por localidad y la lengua hablada. ${ }^{5}$

\footnotetext{
${ }^{3}$ No deja de llamarme la atención que si bien en algunos círculos de la historia de la educación hoy en día ya se da por sentado que la experiencia decimonónica dejó un legado significativo en muchas regiones del país, cuando asisto a coloquios sobre educación en universidades de la Ciudad de México y de los estados, prevalece en muchas ponencias la idea difundida por la propaganda revolucionaria de que las escuelas rurales federales fueron las primeras en atender al campo o a la población indígena significativamente.

${ }^{4}$ La nueva historia política y social del siglo xIx ha estudiado el impacto de los cambios constitucionales que surgen de 1812 en adelante. Véanse, por ejemplo, Guardino (2009) y Thomson con LaFrance (2011).

5 Para estos dos municipios el último padrón que encontré que aún dividiera a la población en "gente de razón" e "indios" o "indígenas" fue de 1874. Archivo de la Junta Auxiliar de San Miguel Tzinacapan, caja 15, Presidencia, doc. 6.
} 
A continuación comentaré la situación de los archivos en Puebla, particularmente para los municipios de la Sierra Norte. En segundo lugar, mencionaré dos temas que pueden investigarse con las fuentes disponibles para 1876-1940. Por un lado, la manera en que se generaban y distribuían recursos para las escuelas y, por otro, la asistencia escolar de niños hablantes de lenguas indígenas (incluyendo las diferencias que podía haber entre cabeceras y pueblos) y el aprendizaje del español.

Por lo general en nuestro país no encontramos una práctica uniforme en cuanto a la conservación de archivos locales pero el historiador tenaz, y con un poco de suerte, podrá encontrar archivos municipales en las cabeceras e incluso archivos de los pueblos ("juntas auxiliares" en Puebla). Hasta ahora, el estado mejor estudiado por la historia de la educación es el Estado de México, que disfruta de un archivo estatal y de archivos municipales con abundante información. En el caso de Puebla el Archivo General del Estado (AGEP) perdió la mayor parte de sus fondos en la década de 1940. A pesar de ello, aún existe en el actual AGEP material útil para la historia escolar en municipios indígenas: la del fondo "Secretaría de Educación Pública; Movimiento de personal y todo lo relacionado con escuelas", que contiene documentación a partir de 1918. En cualquier caso, la mayor riqueza documental para el periodo 1876-1940 en Puebla se encuentra en los municipios. Para los datos que aquí presento utilicé el Archivo Municipal de Cuetzalan, ordenado y mantenido gracias a los esfuerzos de la maestra. Emma Gutiérrez Manzano, así como el Archivo Municipal de Huehuetla, rescatado, entre muchos otros archivos municipales poblanos, por la doctora. Pilar Pacheco Zamudio durante su dirección del Archivo General del Estado de Puebla. También consulté el Archivo de la Junta Auxiliar de San Miguel Tzinacapan (municipio de Cuetzalan), donde la información es muy rica y muchas veces diferente a la del Archivo Municipal de Cuetzalan, a pesar del constante intercambio entre la junta auxiliar y su cabecera municipal. Este archivo fue rescatado hace tiempo por diversos miembros de la comunidad, como parte de las actividades del Proyecto de Animación y Desarrollo, PRADE, A.C., y permanecía en las oficinas de la Junta Auxiliar durante mis últimas consultas en la década de 2010. Todos estos archivos, a pesar de los esfuerzos de quienes los han rescatado, y de instituciones como Apoyo al Desarrollo de Archivos y Bibliotecas de México, A.C. (ADABI), que han colaborado a mantenerlos vivos, se encuentran en una situación precaria, siempre expuestos al deterioro por la humedad o el destrozo de la polilla y los insectos.

Veamos ahora el sostenimiento de la educación. Hasta antes de 1917, las escuelas del estado de Puebla eran sostenidas por los municipios gracias a la recolección de la contribución

\footnotetext{
"Padrón General de San Miguel Tzinacapan. Año de 1874". Sobre el uso del término "indios" e, incluso, acusaciones de "guerra de castas" en Cuetzalan, con base a documentación principalmente del Archivo Municipal de Zacapoaxtla, véase Thomson (1995).
} 
de Chicontepec, un impuesto personal que debían pagar todos los varones mayores de edad (Acevedo, 2015: 53-55). Con estos fondos en los municipios de Huehuetla y Cuetzalan se mantuvieron en el Porfiriato una escuela de niños y otra de niñas en las cabeceras; mientras que los pueblos tuvieron una escuela de niños y algunos de ellos, a partir de la década de 1890, abrieron además una escuela de niñas. Si bien los municipios no recibieron subsidios, tampoco perdieron recursos a favor de zonas más urbanas como ocurrió con los casos de centralización en otros estados. Por ejemplo, en el Estado de México y en Tlaxcala la financiación y administración escolar pasó de manos de los municipios a las del gobierno del estado durante el Porfiriato, desfavoreciendo a las localidades más pequeñas, que vieron cerrar sus escuelas (Bazant, 2002; Rockwell 2007). En el caso de Chiapas, además, la distribución de impuestos escolares fue muy desigual, con las localidades de mayor número de hablantes de lenguas indígenas, y más pobres, pagando más y recibiendo menos (Washbrook, 2012: 158, 166-68). Sería muy conveniente que futuras investigaciones nos hicieran saber qué ocurrió en otros estados con importante proporción de población indígena. ${ }^{6}$ ¿Qué efectos tuvo la centralización de la financiación de las escuelas? ¿Hubo ventajas para las localidades pequeñas e indígenas en los casos en que no hubo centralización, como sugiere el ejemplo del estado de Puebla? El conflicto revolucionario trajo el empobrecimiento de las haciendas municipales, estatales y federales, así como la abolición en 1917 de los impuestos personales que aún sostenían las escuelas en varios estados. En estas condiciones, los estudios que se han hecho para Puebla y Estado de México indican que durante las décadas de 1920 y 1930, a pesar de la entrada de recursos federales, en muchos casos apenas se consiguió igualar la provisión que se había tenido en el Porfiriato (Acevedo, 2015; Alfonseca, 2015). ¿Qué ocurrió en otros estados?

A juzgar por lo ocurrido en Cuetzalan y Huehuetla, las diferencias entre cabeceras municipales y pueblos sujetos, así como entre los pueblos y las localidades menores como barrios y rancherías implicaron jerarquías de recursos, diferencias lingüísticas, así como distintas relaciones interétnicas y políticas, que se reflejaron en la provisión y resultados escolares. Por ejemplo, durante el Porfiriato, era más probable que un niño hablante de lengua indígena asistiera a la escuela en un pueblo determinado, que en una cabecera, probablemente como reflejo de la distribución demográfica y de las relaciones de poder. En la cabecera municipal de Cuetzalan, donde el control político-administrativo de los no indígenas era más fuerte que en los pueblos sujetos del mismo municipio, el número de alumnos nahuas era escaso, normalmente menos de diez alumnos (de un total de 30 a 70 alumnos), a pesar de constituir

\footnotetext{
${ }^{6}$ Por ejemplo, sabemos que las escuelas en Oaxaca pasaron de la administración municipal a la del gobierno estatal en el Porfiriato, pero no se ha estudiado el impacto de este cambio en términos de la cobertura escolar (Traffano, 2007).
} 
la población nahua la mayoría (en 1900, 63 por ciento de la población del municipio hablaba el náhuatl) (Dirección, 1902). Por el contrario, en el pueblo sujeto de San Miguel Tzinacapan, donde todas las autoridades eran nahuas, y solamente el secretario y el maestro eran no indígenas, se alcanzó una asistencia de alrededor de 90 alumnos, todos indígenas. El ejemplo de la cabecera municipal de Huehuetla parece confirmar que la mayor presencia y poder de los no indígenas en una localidad estaba relacionada con la menor presencia de los indígenas en la escuela. En Huehuetla, donde el control político-administrativo de los no indígenas era más débil que en Cuetzalan, más niños indígenas asistían a la escuela de la cabecera tanto en números absolutos como porcentuales. ${ }^{8}$

Por otra parte, los archivos municipales de Huehuetla, Cuetzalan y Zacapoaxtla, así como el archivo de la Junta Auxiliar de San Miguel Tzinacapan, permiten identificar algunos problemas de lecto-escritura en estas escuelas públicas que tuvieron el castellano como lengua de instrucción. Durante el Porfiriato no hubo una política explícita de enseñanza del castellano como segunda lengua, y tras la Revolución, aunque sí se discutió este asunto, siguen apareciendo pistas poco estudiadas de las múltiples dificultades enfrentadas en las aulas para la lectoescritura (Acevedo, 2008 y 2011). Otros archivos locales, así como los generales de los estados, pueden arrojar información en este sentido además de permitirnos estudiar el contexto más amplio, por ejemplo, cómo se relacionaba la situación socioeconómica con la inscripción y asistencia escolares, y con la alfabetización; o cuál fue la preparación, reclutamiento y permanencia de los maestros. Para el siglo xx también será muy útil la historia oral puesto que ésta puede darnos pistas que solo muy de vez en cuando aparecen en archivos, por ejemplo, sobre la cuestión de si los maestros conocían la lengua indígena de la región y la usaban para facilitar el aprendizaje (Castillo, 2017). ${ }^{9}$ Asimismo, para una cabal compresión de la lectoescritura en estos contextos, nos hace falta conocer su papel en espacios no escolares. Ya contamos con algunos trabajos que muestran la manera en que una educación incipiente en la escuela podía ser luego complementada con la experiencia en el gobierno local o en el mundo laboral (Bertely, 1998; Rockwell, 2018: 601-691, 729-756; Escalante, 2014).

No es posible aquí hacer un recuento exhaustivo de la riqueza de los archivos locales. Además de los ya mencionados, hay datos sobre los libros y materiales disponibles en las aulas, las materias cursadas, la realización de exámenes públicos y la celebración de fiestas

\footnotetext{
${ }^{7}$ Los datos se refieren a varones. El número de niñas en las escuelas fue demasiado pequeño para hacer comparaciones significativas (Acevedo, 2018).

${ }^{8}$ La menor penetración de no indígenas en el municipio de Huehuetla se refleja en la situación lingüística: en 1900 el 86 por ciento hablaba el totonaco (comparado con 63 por ciento de hablantes de náhuatl en el municipio de Cuetzalan). Censo General de la República verificado el 28 de octubre de 1900 conforme a las instrucciones de la Dirección General de estadística, México, 1902.

${ }^{9}$ Rockwell (2018: 601-630) ha combinado muy exitosamente entrevistas con documentación de archivos.
} 
cívicas. El papel de los padres de familia puede rastrearse con más facilidad que el de los estudiantes. Todo ello permite analizar cómo procesos escolares específicos como la introducción de la escuela graduada, o de la simultaneidad de la enseñanza de la lectura y la escritura, contribuyeron a la escolarización, la formación de subjetividades, de ciudadanos, así como a construir formas y procedimientos de Estado, susceptibles de control administrativo centralizado. La lista podría continuarse, ojalá sepamos sacar provecho de los archivos disponibles y rescatar los que están en riesgo de desaparición.

\section{Referencias}

Acevedo Rodrigo, Ariadna (2008), "Ritual Literacy. The Simulation of Reading in Rural Indian Mexico, 1876-1930”, Paedagogica Historica, vol. 44, núm. 1-2, pp. 49-65.

Acevedo Rodrigo, Ariadna (2011), "La ignorada cuestión del idioma: La educación en los pueblos indígenas de Puebla, México, 1876-1930" en Lourdes Alvarado and Rosalina Ríos (eds.), Grupos marginados de la educación, siglos XIX y XX. Instituto de Investigaciones sobre la Universidad y la Educación (IISUE) de la UNAM, México, pp. 431-468.

Acevedo Rodrigo, Ariadna (2015), "Entre el legado municipal y el avance del gobierno federal: las escuelas de la sierra norte de Puebla, 1922-1942", Relaciones, núm. 143, pp. 51-84.

Acevedo-Rodrigo, Ariadna (2018), "Liberalism and Revolution at the Grassroots. Indians, Education, and Municipal Government in Puebla, Mexico, 1875-1940" (manuscrito).

Alfonseca Giner de los Ríos, Juan (2015), "La federalización de la enseñanza. Concurrencia escolar y alianzas sociales en contextos rurales del oriente del Valle de México, 1922-1947", Relaciones, núm. 143, pp. 11-50.

Bazant, Mílada (2002), En busca de la modernidad: Procesos educativos en el Estado de México, 18731912, Colegio Mexiquense, México.

Bertely, María (1998), "Historia social de la escolarización y uso del castellano escrito en un pueblo zapoteco migrante", tesis doctoral, Universidad Autónoma de Aguascalientes, Aguascalientes, México.

Bustamante Vismara, José (2014), Escuelas en tiempos de cambio: politica, maestros y finanzas en el valle de Toluca durante la primera mitad del siglo XIx, El Colegio de México, México.

Castillo Molina, Daniel Gibrán (2017), "El idioma mexicano visto desde las escuelas de los pueblos tlaxcaltecas, 1900-1930", Revista Mexicana de Historia de la Educación, vol. 5, núm. 10, pp. 181-197.

Cedeño Peguero, María Guadalupe (2013), "Religiosos y educación. Métodos y enseñanzas en el antiguo obispado de Michoacán", Revista Mexicana de Historia de la Educación, vol. I, núm. 1, 29-58.

Chowning, Margaret (2017), "Culture Wars in the Trenches? Public Schools and Catholic Education in Mexico, 1867-1897", Hispanic American Historical Review, vol. 97, núm. 4, pp. 613-649.

Civera, Alicia (2008), La escuela como opción de vida: la formación de maestros normalistas rurales en México, 1921-1945, El Colegio Mexiquense, México.

Dirección General de Estadística (1902) Censo General de la República verificado el 28 de octubre de 1900 conforme a las instrucciones de la Dirección General de Estadística, México.

Escalante, Carlos (2003), "Indígenas y educación en el siglo XIX mexicano" en Luz Elena, Galván Susana Quintanilla y Clara Inés Ramírez (coords.), Historiografía de la educación en México, comie, México. 
Escalante, Carlos (2014), Mazahuas, campesinos y maestros. Prácticas de escritura, tierras y escuelas en la historia de Jocotitlán, Estado de México (1879-1940), Colegio Mexiquense, México.

Flores Cordero, Karina (2017), "El doble uso de un camión escolar. Representaciones e identificación en la Escuela Regional Campesina de Ayotzinapa (1935-1938)", Revista Mexicana de Historia de la Educación, vol. 5, núm. 9: 95-116.

Giraudo, Laura (2014), "No era un desierto. La Secretaría de Educación Pública y la educación rural en el estado de Veracruz, periodo posrevolucionario" en Luz Elena Galván Lafarga y Gerardo Antonio Galindo Peláez (eds.), Historia de la educación en Veracruz. Construcción de una cultura escolar, Universidad Veracruzana y Secretaría de Educación del Estado de Veracruz, México, pp. 171-96.

Guardino, Peter (2009), El tiempo de la libertad. La cultura política popular en Oaxaca, 1750-1850, Universidad Autónoma Benito Juárez de Oaxaca, Oaxaca.

Manzanilla Dorantes, Juan Ramón (2015), "Espita. Federalización educativa, concurrencia y conflicto, 1922-1935", Relaciones, núm. 143, pp. 103-127.

Martín Alcaraz, Alicia y Carlos Escalante (2017), "Prácticas educativas, 'el problema de la lengua' y usos indígenas de la lectura y la escritura en la historiografía de la educación del siglo xIx en México", Revista Mexicana de Investigación Educativa, vol. 22, núm. 73, pp. 347-366.

Martínez Pineda, Idunaxhíi (2014), "Cultura escolar en las escuelas primarias de Juchitán", tesis doctoral, Universidad Autónoma del Estado de Morelos, Cuernavaca, México.

Rockwell, Elsie (2007), Hacer escuela, hacer estado. La educación posrevolucionaria vista desde Tlaxcala, El Colegio de Michoacán, CIESAS y Cinvestav, México.

Rockwell, Elsie (2018) Vivir entre escuelas. Relatos y presencias. Antología esencial, CLACSO, Buenos Aires.

Roldán Vera, Eugenia (2014), "La perspectiva de los lenguajes en la historia de la educación", Ariadna histórica. Lenguajes, conceptos, metáforas, vol. 3, pp. 7-14.

Sigüenza, Salvador y Graciela Fabián (2013), "Historia de la educación indígena en la configuración del Estado nacional y la ciudadanía en México" en María Bertely, Gunther Dietz y María Guadalupe Díaz (eds.), Multiculturalismo y Educación, 2002-2011, Consejo Mexicano de Investicagación Educativa (COMIE). Colección Estados del conocimiento, México, pp. 81-115.

Staples, Anne (2005), Recuento de una batalla inconclusa: La educación mexicana de Iturbide a Juárez, El Colegio de México, México.

Tanck, Dorothy (1999), Pueblos de indios y educación en México colonial, 1750-1821, El Colegio de México, México.

Thomson, Guy (1995), "Francisco Agustín Dieguillo: un liberal cuetzalteco decimonónico (1861- 1894)" en Jane-Dale Lloyd y Laura Pérez Rosales (eds.), Paisajes rebeldes. Una larga noche de rebelión indígena, Universidad Iberoamericana, México, pp. 77-148.

Thomson, Guy con David LaFrance (2011), El liberalismo popular mexicano. Juan Francisco Lucas y la Sierra de Puebla, 1854-1917, Ediciones de Educación y Cultura y Benemérita Universidad Autónoma de Puebla, Puebla.

Traffano, Daniela (2007), "La creación y vigilancia de las escuelas municipales 'continuará a cargo de las autoridades [...]' Ciudadanía, escuela y ayuntamientos" en Escobar, Antonio (ed.) Los pueblos indios en los tiempos de Benito Juárez, UABJo/UAM, México, pp. 69-90.

Traffano, Daniela (2016), "Por la muy merecida importancia que la enseñanza ha obtenido..." Documentos sobre instrucción pública en Oaxaca a finales del siglo XIX, Carteles Editores, Oaxaca.

Vaughan, Mary K. (2001), (e.o. 1997) La politica cultural de la Revolución, maestros, campesinos y escuelas en México, 1930-1940, FCE, México.

Washbrook, Sarah (2012), Producing Modernity in Mexico: Labour, Race and the State in Chiapas, 1876-1914, Oxford University Press, Oxford. 